\title{
KRITIK UND ERKLÄRUNG DES WINSBEKEN UND DER WINSBEKIN.
}

Die folgenden studien beschäftigen sich mit zwei lehrgedichten des mittelalters, von denen das eine wegen seiner poetischen schönheit, die im einzelnen zu erörtern hier nicht der platz ist, und seiner culturgeschichtlichen wichtigkeit fur die erkenntnis der grundsätze ritterlicher moral in der blutézeit des höfischen lebens unser volles interesse in anspruch nimmt und das andre, wenn auch an innerem poetischen werte mit jenem ersten nicht von ferne vergleichbar, doch auch wegen seiner nahen formellen wie inhaltlichen verwantschaft mit ihm der beachtung und untersuchung wert scheint: Winsbeke und Winsbekin. Zu einer neuen constituierung des textes beider gedichte, die ich demnächst zu veranstalten gedenke, sollen die betrachtungen der folgenden capitel gewissermassen den weg bahnen, indem sie einige vorfragen der kritik und erklärung behandeln. Nicht wertlos wird es erscheinen, dass ich diesen untersuchungen zuerst eine collation einer von Haupt ftr seine ausgabe beider gedichte (Leipzig 1845) noch nicht benutzten, wenn auch verhältnismässig jungen handschrift vorausschicke.

I. Strophen des Winsbeken und der Winsbekin in der Kolmarer handschrift.

Zu den handschriften, welche Haupt zu seiner kritischen herstellung des textes benutzte, kommt noch die Kolmarer liederhandschrift, nach mannigfachen schicksalen jetzt im besitz der königlich bayrischen hof- und staatsbibliothek zu München als deren cod. germ. 4997. Ueber die handschrift im allgemeinen bat Bartsch in der einleitung zu seiner ausgabe der 
meisterlieder (Stuttgart 1862) gehandelt, woselbst sich auch ein genaues inhaltsverzeichnis derselben befindet. In dieser handschrift nun (ich nenne sie im folgenden, da Haupt den buchstaben $K$ schon an die Basler handschrift vergeben hat, $k$ ) finden sich an verschiedenen stellen strophen des Winsbeken und der Winsbekin, darunter manche doppelt und nicht ohne discrepanzen. Bartsch hat in der erwähnten einleitung die abweichenden lesarten dieser strophen teils unvollständig, teils nicht mit voller genauigkeit, teils endlich gar nicht angegeben (vgl. Meisterl. s. 75. 82). Für das folgende variantenverzeichnis, in dem alle abweichungen vom Hauptschen texte sich finden, soweit sie nicht rein orthographischer oder dialektischer natur sind, stand mir eine vollständige genaue abschrift der strophen zur verfugung, die herr dr. H. Schnorr v. Carolsfeld in Munchen selbst fur mich anzufertigen die freundlichkeit hatte, wofür ich ihm auch an dieser stelle meinen herzlichen dank sage.

Blatt 728 a. tuberschrift: in der griss wyse dez tugenthafften schrybers. Es folgen 48 strophen des Winsbeken. Varianten: 1,3 den wolt er. 4 sprach ym myn kint du. 5 alle arge list. 6 alz du selber. 8 myn lieber son daz ist. 9 ein andrer (oder anderr? ein häkchen bedeutet in der handschrift bald er, bald re). $\mathrm{Zu}$ dieser strophe eine melodie. - 2,1 mynne ynneclichen. 2 kan dirs. 4 dü sichst; gockel. 5 regen. 6 jungst sin. 8 swinde. 9 vnd wer ich noch vil dienen. 10 daz ist. - 3,2 brendet. 3 dir aüch also. 4 von tag zü tag von jar zü jar. 5 sinne vor. 6 richt din leben vor hin so. 7 din sele. 8 wie; ist din. 9 dann also. 10 vor. $-4,3$ ander leben. 5 vil me wann. 6 niltii nü heuffen. 8 vor. 10 daz dir verslahen icht werd. 5,1 alle. 2 hercz und synne bedenken. 3 wer hat. 9 in dinen. 10 lass. - 6,2 myn sin. 4 bring es; grüben. 5 dirr gewin. 7 got soltü billich ern an yn. 8 wercke krümp. 9 den worten. 10 den wercken; ald. - 7,2 trügen den paffen. 4 ich. 5 fürr. 6 sin mit dräuwen holt. 10 lichnam. - 8,1 dir füg; byderb wip. 2 nach gottes lob. 3 han. 4 also stee. 6 dar yn. 7 waz wiltü wunne haben me. 8 beschicht in dren) (mit spiritus asper über dem $w$ ) pflet. 9 irn samen. - 9,1 synniclichen trag. 3 din tügent; sag. 4 waz. 5 lass nit verhergen dich. 9 werren zwschen; tragen. 10 vnd daz ist iudas heübt geschicht. - 10,1 wer. 2 in nit. 3 wer. 4 mit scham vber den. 5 der güte. 
7 frawen allen wole. 8 und ist ir heynen eren fry. 9 do by. 10 und fehlt; wone. $-11,4$ von sünden. 5 ein wünnenbernder. 6 von dem. 7 czüchte noch der scham. 9 einer syn. - 12, 1 ein nunnenberndes. 3 ein freüd der welte züuersicht. 4 wiss man. 5 heyt. 7 folliclich vnd breyt. 9 beschüff. 10 liess vor engel. - 13,1 kanst nit genissen. 4 daz dü yn gist din liebe zit. 5 vnd daz dir güte. 10 myn getrosten din. $-14,3$ lass dir. 5 oder lang. 7 ane wang. 8 mit wirdekeit betagt. 9 dryack daz eyter. 10 dir leyt verjagt. - 15,1 sag dir. 6 reyner wibe. 10 als ein wint. - 16, 2 dü yn ir lobes czil. 3 und dien. 4 dester. 6 gegeben. 9 komet zü lon. 10 vol ym der dar an ligen soll. $-17,5$ dir nit verhil. 6 der welte. 9 der vmb. 18, 1 last; leben. 3 so merck waz er dir freude git. 4 balde folgen (am rande nachgetragen). 5 gramer vch. 7 in daz. 9 dir noch den selben. 10 ym hercz und müt. - 19,3 getrew dar zü kün vnde slecht. 4 ist. 5 vor. 9 willü yn dann. 10 vil bass. - 20,3 erdenck. 4 vergalt. 5 doch swent ir myn recht als der walt. 7 myn hat vil mangen. 8 verwegen. 10 fraw selde; dez segens. - 21,1 gein. 2 senck vil schone. 3 pecht (sic) als er sy. 4 lass an din ernst mit diner meysterschafft. 5 baz vnd ye bass; dyne krafft. 6 zün. 8 oder. 9 güttes ritters. 10 vff der süst. - 22,4 enweiss. 5 rechtem. 6 dem. 7 wunne me. 8 der güten. 9 so ein. - 23, 2 vernym eben waz ich lere dich. 4 aldarnach er. 5 rech; zu vil nit sprich. 6 mere zü. 7 von dem. 8 die oren. 9 smeicheleren. $-24,2$ vz deme anger. 3 sit. 6 gezomet sie rechte dinen zorn. 10 verdienet. - 25, 2 danne eins. 4 munde hyn. 10 dü lebest. 26, 1 nit (uber der zeile) wer zü blicke füge entnymet. 3 geriben. 5 daz lieht gewant daz ist. 6 daz hindan lühtet cleydet wol. 9 alsüs wolle driegen sich. 10 dar vnder. - 27, 2 swellet. 3 düt auch. 4 sonder qüal. 5 selden flüssig. 6 etwenne. 7 von eyme züm andern alz. 9 nit sanffte; yme. 10 sine. - 28, 2 den wiben. 3 kiesent. 4 korn fehlt. 5 der ist hochgeborn. 7 erkorn fehlt. 8 der alle zit der eren gert. 9 fur der sich helt sonder. 10 vnd hüre boser da ist dan fernt. - 29, 1 got. 2 also daz er yt lige obe. 3 benympt ez; synne. 5 güt ist. 6 lieber ist danne; weme. 8 wen. 9.10 der verzihet sich der beider ee danne er auch daz eine ferlür. - 30,1 dine. 4 gehem; daz ist. 8 wurff. 9 forhte. - 31, 1 
waz. 3 zü aller. 4 würt din lop din wirde. 6 daz fehlt; der argen bosen. 9 die in kranckem gemüte. 10 sie von art sint geslachat. - 32, 1 der fogel der ee danne zü rechter zyt. 2 syme. 3 selber; git fillicht. 4 zü eyme. 5 der rede. 6 des an daz. 8 müstü leben an eren lan. 9 dir fehlt. 10 dü hettest nie. - 33,1 habe. 2 daz la. 3 wer ye gerne. 9 dez; alz. 10 einen dregen. $-34,5$ wer. 8 sit dz die rede zweyget sich. - 35,1 wer; selber. 2 getrünez. 3 ferlüret; rede. 4 eyme; dogendenden (sic). 5 wer; redet. 6 dz mans nit fur güt enhat. 7 verlüret sinen willen. $8 \mathrm{wz}$ froüdes frünt. 9 er wil als selber stüren. 10 rechte als in eine bach. - 36,1 alie fehlt. 2 zü der. 4 in deme. 6 din drost myn drost eine an mich komen. - 37, 3 syge. 6 ist feblt. 7 deme; solliche. 9 sige; oder frome. 10 do kan er küme komen von. - 39, 1 wort. 5 wider nyid noch hass. 6 gegen; hochgemüt. - 40,1 hoffart. 1 yme susse. 5 fehlt. 7 zür; dort der bitter. 8 wer fünden in den schanden wirt, und fert. 9 deme; syme. - 41, 1 lange zit. 2 wer; hoffart. 3 im fehlt. 4 daz da verfellet. 6 rehte fehlt. 7. 8 umgestellt. 8 wer. $-42,1$ willü; guot fehlt. 3 jüngen mannen. 5 sol. 7 streben. 9 löuffel; wise. 10 kalzen. - 44, 1 gan an. 7 wanne ez ist grosse missetad. 8 dar zü; fründez. 9 schlüss. 10 din zünge. - 45, 1 beyde. 2 selen vol. 3 wer. 5 wer. 7 in der. 8 ner so verlüret. - 50,1 wer. 3 wanne er hat schanden ane gesiget. 4 fehlt. 5 krüch er ioch. 6.7 umgestellt. 6 des hun ich ye mit willen gert. 8 worzelen. 9 eyme. 10 vom. - 52,1 zwey ding eren. 5 daz edel gestein. 6 alz dünt war. 9 war. 10 schrencket hin. - 53, 2 gestercket mit. 5 wer. 7 yme. 8 verdienet. 10 den klam. 55,2 ir strosse ir steige ger du nit. 4 wie gut geferte. 5 dich ez ist. 6 wo. 7 pore. 10 befriden. - 56, 1 enwil; me. 3 sin alles. 4 rechten allen dryn. 5 deme. 9 dusent nement lere von.

Blatt 732 a. uberschrift: $X V$ lieder in dem selben done. Es folgen 15 strophen des Winsbeken. Varianten: 65, 1 din hohen. 2. dinen. 4 des mane zweimal. 6 steten rünen. 7 von schülden. $8 \mathrm{daz}$; myn lip gesondet. 9 ein phant. - 66, 1 here. 2 fervallen. 8 dogende. 9 schecher. 10 fehlt. $-67,1$ wingart. 2 vnd. 6 nü; der alter. 7 in sine stricke mich. 10 den mir. - 68,1 noch trostet mich gedinge wol. 2 daz; endnlich für 
war. 3 dien ich 4 am lon. 6 dagemercker. 7 wircken. 10 myn lon. - 69, 1 vnd. 2 vnd. 3 obe. 7 die rechter rüne ye helffe bot. - 70,2 gebürt eins tages. 4 würt. 5 da hat din worheit me gewalt. 7 daz ich ein teyl nie engalt. 10 grosser schülde. - 71, 1 alle. 2 kein. 3 von oben abe biz üff den grünt. 4 sige. 5 dine. 6 vertümst. 8 zu büssen. $9 \cdot$ sihst; mynem. - 72,2 mir nach. 3 la mich hie also lange. 6 liess. 7 dine. 9.10 umgestellt. 9 welte lebe. $-73,3$ stärckem rüwen. 5 der fehlt. 9 sie. 10 din fehlt. $-74,3$ wammen. 10 herbarmhertzikeil. - 75, 2 die nydert hohert wen sie wil. 3 nabächodonisor. 4 hette. 5 hoffart. 9 den dinen zorn den ich verdient. 10 da her han do vor mich bewar. - 76,1 gesagen. 2 biss her. 4 swarem. 5 were ir. 6 wie; sonden enteylt. 7 wonet in ir. 8 beklieben. 10 fant ich an; geschrieben. 77, 2 rechl als ein sonder. 5 mag hende oder füss. 6 geregen: dich fehlt. 8 van hymel. $-78,6$ mich sere. 10 er dinen willen. - 80, 3 lass ich. 4 vnd. 5 büwet und. 7 han es. 9 eingeborn son. 10 ziehen wollen.

Blatt 800 a. uberschrift: der getriuven muter lere in der gruiss wyse dez tugenthafften schrybers. Es folgen 8 strophen der Winsbekin. Varianten: 1,2 zu ir; schon. 3 die ic. 4 geheiliget; der liebe. 5 erst. 7 wyser lere. 8 ist des. 9 soln; hôhe feblt; loben drum. - Vor 2: die tochter. 2,1 volg; liebe. 2 loben in so best ich. 4 seh mit augen. 5 sin tugend. 6 wil in sinen. 7 selb der eren. 8 vnd; soln ir kint. Statt 9. 10: eren vnd sie sie lern die in gehorsam sint. - 4, 1 nu rat lieb. 4 woll. 5 wil sin fro und fry. 6 herwegen. 7 hoffarl vnd eren auch der zwey. 9 welch fraw; kumpt. 10 sich dez verslichlet. - 5,2 vnd dar vnder in. 3 wirt. 4 crancz von rosen. eben. $5^{*}$ den ergern solt mit zuchten geben. 6 lass in din hercze scham vnd mass. 7 hilff aller vnzucht wyder steben (sic). 8 so macht vermyden stete pin. 10 wo bose. - 6,1 mass daz. 2 die fehlt; geben. 3 let sie got leb in diner. 4 diner. 5 du mach yn eren. 6 nu rat du liebe muter myn. 7 solicher ding bin ich nit wys. 8 waz wilder. 9 wie vnde wo ich myden sol. 10 mache nit. - 7,2 hoff genysel. 3 wo ein. 4 lar sie die auge fliehen hien. 5 also sie hab. 6 gesche. 7 ir lop mit krancken gwin. 8 mercker; vns gesicht. 9 zwing dine. 10 das nit ich kint und bittens dich. $-8,1$ muter dir sy vor war ge- 
sagt. 2 wie clein ich hab. 3 die fraw mir nymmer wol behagt. 4 welch. 5 her hebet recht alz. 6 ond auch dar vnder lachent vil. 7 die prysent nit den yren gral. 8 ouch feblt; vnfraulich müt. 10 irr gebort vil dicke. - 9,1 wyse. 2 sint din. 3 werck. 5 nest vogel. 6 der gyt den andern sich zu spil. 7 vnd wirt sin federn ym verzogen. 8 ein uss flug der ist nit zu loben. 9 kint dir mag auch also geschehen. 10 halt du dich in der eren cloben.

Blatt 803 a. uberschrift: in der griiss wyse des suines antwurt vff des vatters lere die er vor vnd nach hat getane dem sone. Es folgen 11 strophen des Winsbeken. Varianten: 57, 1 velterlich. 5 die alle. 6 wol gemessen hohe. 7 die bitt ich sie mich gutes man. 9 mir dort wolle (getilgt: darob). 10 riche darumbe (getilgt: musse). - 58,1 ach vatter ich bin noch ein kint. 2 doch sehen ich daz die welte birt. 3 ein gogel werck ir freude sint. 4 recht alz ein kol verbrynnen wirt. 5 ein mist ir trost ein vnyenyst. 6 let; frund in swerer hab. 7 ynnen. 10 hab. - 59, 1 vater fehlt; ein alter; mude. 2 hab. 3 wer ie. 4 nahe leyten; stab. 5 sich vatter da gruwet mir ab. 7 volget zu dem grab. 9 wysheit ist vernicht. 10 selbes. - 60,1 eim wysen; schône fehlt; zympt. 2 schon daz; tuo foblt. 3 nympt. 4 da worde sust vil licht vermitten. 5 wol alt. 6 er nit bekennet. 8 der toren. 9 lob. 10 biss ans ende. $-61,1$ vrlaub; ich zeln. 2 gein dir entsliessen gar. 3 es; by mir verheln. 4 vor. 5 leg. 6 selber. 7 in siner. 8 hab sol; zeln. 9 vor. 10 hymel rich. $-62,1$ die red. 2 reyner. 3 fraun. 4 so vil von gotte. 7 vmb dich. 8 gelebt. 9 geben. - 6, 1 leb; hab. 3 nieman ab. (1) enruch dich. Im ubrigen wie oben. - 7, 1 sytt. 3 selber mit. 5 furebas. Im tubrigen wie oben. - 63, 1 was. 2 gein dysen. 3 sint. 4 sund. 5 sag. 6 keuffen. 9 wirt. 10 williclich gegeben. - Hierauf eine strophe, die allen andern handschriften fehlt:

Nu sag an mynn ich frage dich wie stet din hilff vnd din gewalt sie sint verdorben duncket mich vnd sint auch nit alz ee gestalt sie jehent du seist ein teil zu bald. nu scham dich durch die reine wyp. daz vaverswendet stet der walt 
din schappel dir vneben stat

daz hat gemacht ein nuwer walt

daz gut weyss got nu vor uch gat.

Bartsch hat diese strophe (Meisterl. s. 82) in correctem mhd. hergestellt, gibt jedoch in zeile 9 statt walt site, offenbar conjectur. - 64, 1 suss; musten wangen. 3 tut uch. 4 volgent. 6 dann. $8 \mathrm{ez}$ ist nit gar ein kindes spil. 9 wer; arbeit. 10 gotte dienen wil.

Blatt $803 \mathrm{~d}$. uberschrift: ein ander lere dez vatters; In der gruiss wyse. Es folgen 27 strophen des Winsbeken. Varianten: 22, 2 zu hoff. 5 trag. 7 selde. - 23 ganz wie oben. - 24, 3 vnerwegen. Sonst wie oben. $-25,4$ zü gehet. Sonst wie oben. - 26. $27 \mathrm{ganz}$ wie oben. - 28,4 korn. $10 \mathrm{da}$ fehlt. Sonst wie oben. - 29, 2 daz es. Sonst wio oben. 30 ganz wie oben. $-31,4$ din wurde. 10 geslaht. Sonst wie oben. - 32, 2 nesten. 9 besser dir. Sonst wie oben. $-33,8$ gar fehlt. Sonst wie oben. -34 wie oben. $-35,3$ gar güter. 4 dogenden. Sonst wie oben. - 36. 37 ganz wic oben. 39,5 weder. Sonst wie oben. - 40 ganz wie oben. $-41,4$ ein spil. Sonst wie oben. - 42, 3 jüngen manne. Sonst wie oben. - 44,6 so nü. Sonst wie oben. - 45. 50 ganz wie oben. - 52,6 also. Sonst wie oben. - 53 ganz wie oben. 55,10 gefryden. Sonst wie oben. $-56,9$ von dryn. Sonst wie oben.

Blatt $806 \mathrm{~b}$. uberschrift: item $X V$ lieder aber in dem selben done. Es folgen 15 strophen des Winsbeken. Varianten: 65,4 des mane. Sonst wie oben. - 66, 3 fryen. Sonst wie oben. - 67,9 nem. Sonst wie oben. - 68,6 dagewercker. Sonst wie oben. - 69 ganz wie oben. - 70,5 me gestalt. 7 des ich. Sonst wie oben. - 71 ganz wie oben. - 72, 2 noch. 10 dan. Sonst wie oben. - 73. 74 ganz wie oben. 75, 3 nabüchodonosor. Sonst wie oben. $-76,4$ swaren. 10 deme. Sonst wie oben. - 77,6 ich dich. 8 von himel fehlt. Sonst wie oben. - 78, 3 an rüven. 10 und in din. Sonst wio oben. - 80 ganz wio oben.

Den dialekt des schreibers der Kolmarer handschrift, der auch dichter war (vgl. Meisterl. 8. 186), im zusammenhang zu untersuchen wurde eine dankbare aufgabe sein. Was unsre strophen anbelangt, so ist hier der dialekt kein reiner: es sind 
eine menge von formen aus der ostfrănkischen vorlage in einen andern fränkischen dia!ekt, jedenfalls den rheinfränkischen, umgesetzt, aber auch sehr viele unverăndert stehen geblieben. Die kleine zusammenstellung, die ich bier gebe, macht auf vollständigkeit keinen anspruch, sondern enthălt nur das eigenartigste der lautlichen verhältnisse. Eine vollstãndige untersuchung wurde auch alle kleinen eigenheiten der ortbographio in $\mathrm{ihr}$ bereich $\mathrm{zu}$ ziehen haben.

Vocalismus. Statt $a$ erscheint hăufig $\hat{o}$ : ône 1,5. Wkin 7,6. 8,9. wôrheit 17,5. 70,5. wôrhaft 56,8. wôr 52,6. nôhe 30,5 . 56, 5. nôch 34,6 . 42, 7. rôt 35,2 . mòze 31, 1. 41, 6. 45, 3. 56, 2. wôge 42, 4 . strôze 55,2 . spôte 68,7 . slôfen 42, 10. verslôfen 68,9 . siôt 70,6 .

Statt $u$ erscheint häufig $o$ vor einfachem nasal oder nasal + consonant: son. vrome 37,9. gebonden 53,3 . wonder $74,2$. wondern 76, 2. vonden 40,8 . 73,9 . sonde $66,2.69,4$. 70, 3 . 71, 7. 73,5 . 74,7 . $76,3.77,4$. sonden 65,8 . sonder $69,3.71,6$. 77, 2. Ferner steht 0 in worzelen 50,8 und hăufig in jogent, togent.

Consonantismus. Hier interessiert vor allem der stand der lautverschiebung. Folgende formen weichen vom normalen mbd. stande ab. Labiale: paffe 6,6. 7, 2. - plegen 20,10. 24, 1. 50, 1. - brîs 18, 9 .

Dentale: $\operatorname{dag} 68,6 .-$ dal 45, 7. - gidikeit 29,5. - diuvel 40,3. - doben 29, 7. - dogent 22, 3. 28, 3. 5. 9. 30, 6. 31, 8. 35, 4. 50, 1. 65, 10. 66, 8. 69, 6. 71, 5. - dopelspil 20,9. - dôre 5, 7. 37, 1. - dôt 53,6. - draege 33, 10. - dragen 22, 5. 23, 6 . 33,1. 39,5. - dranc 14,2. - dreten 44,10. 59,3. 67, 5. driegen 26,9. - drinitatt 65, 1. - driuve 7, 6. 8, 8. 16, 7. 39, 3 . - drôst 36,6. - dump 6,10. 17,8. 32,4. - duon 23, 8. 27, 3. 30,6. 36, 3.8. 39, 2. 52,6. 74,6.9. 77, 1 (doch missetad 30,7. 34, 4. 44, 7. 65, 3. getaden 80, 2). - disent 56,9. - zwing Wkin $7,9$.

Hãufig ist vor ein anlautendes $e$ ein $h$ getreten: herbarmen und verwante worte 10,4.5.6. 74, 10. - herkennen 11, 8. 17,9. 19, 2. - herholn 36,5. - herweln 61,10. - herwinden 63, 7 . - herwegen Wkin 4,6. - herzogen 8,9. - herheben 8,5.

Verwechselungen von $d \hat{o}$ und $d \hat{a}$, diu und die sind hăufig. 
Es erubrigt noch auf die frage nach dem verfasser unsres gedichtes einzugehen, da uns hier unsre handschrift auf den ersten blick sehr merkwürdiges bietet. Die handschriften mit ausnahme von $\mathrm{C}$ geben alle das gedicht ohne verfassernamen, B sogar ganz ohne uberschrift: $\mathrm{C}$ gibt von Winsbach. Dass dies der name des dichters ist oder vielmebr zunächst nur, dass der sammler oder schreiber von $\mathrm{C}$ einen Winsbach fur den dichter hielt, als den dichter wusste oder in seiner vorlage angegeben fand, unterliegt keinem zweifel. Wenn wir auch sonst von einem solchen dichter nichts weiter wussten, so war es doch nichts weniger als kuhn in dem Windesbecke einer stelle des Renners denselben mann zu seben. Die stelle lautet (MSH 4, 872. Gottfr. v. Neif. V. Haupt):
gîtikeit, luoder unde unkiusche,
muotwille unde unzemlich tiusche
habent mangen hern alsô besezzen, daz sie der wîse gar hânt vergezzen, in der bie vor edele herren sungen, von Botenloube und von Môrungen, von Linburc nnd von Windesbecke, von Nife, Wildôn and Brânecke.

Meiner uberzeugung nach würde diese erwăhnung bei Hugo von Trimberg allein beweisen, dass es einen dichter des namens gegeben habe, den auch die handschrift $C$ fur unser gedicbt angibt, wenn auch weitere urkundliche zeugnisse fur das geschlecht sich nicht fänden (Haupt vorr. XII. Zs. fda. 15, 261). Der einzige, der an dem Winsbeken als dichter zweifelte, war Goedeke (Grundr.1 1, 42), gegen den jedoch Pfeiffer Haupts deductionen in schutz nahm (Germ 2,501). Nach unsrer Kolmarer handschrift nun wäre das gedicht in der grussweise des tugendhaften schreibers abgefasst. Ist auf diese angabe hin der schreiber als verfasser anzunehmen? Denn zunăchst muss festgehalten werden, dass, wenn wirklich die Winsbekenstrophe eine weise des schreibers gewesen wäre, wovon anderweitig nichts bekannt ist, der schreiber darum noch nicht notwendig der verfasser zu sein braucht. Bartsch (Meisterl. s. 158) und Goedeke (Grundr.2 1, 162) scheinen geneigt ihn dafur zu halten, Bartsch mehr zurlickhaltend und zweifelnd, Goedeke offener und bestimmter. Ueber den tugendhaften sehreiber ist zu vergleichen MSH 4, 463; J. Grimm und 
Haupt, Zs. fda. 6, 186; Funkhänel, Zeitschr. f. türing. gesch. 2,195; Lachmann, Klein. schr. 1, 316. Ganz abgesehen davon, dass der schreiber durch seine beteiligung am Wartburgkriege etwas in ein mystisches dunkel gehtillt ist, findet sich auch in seinen sonst erhaltenen gedichten (MSH 2, 148), von denen v. d. Hagen 4, 467 eine schöne charakteristik gibt, nichts, was entfernt an die art des Winsbeken erinnerte: einzig das streitgedicht zwischen Keie und Gawein könnte man heranziehen, das ihm vielleicht nicht einmal gehört. Die minnelieder des schreibers sprechen eine idyllische, zuweilen elegische natur aus, versenken sich in die empfindung, lassen jedoch kaum eine recht greifbare individualität erkennen: von den hohen ethischen gedanken und dem plastischen, gedrungenen, zuweilen an Wolfram erinnernden stil des Winsbeken finden wir bier nichts. Was Goedeke noch als argument fur seine annahme auffuhrt, man musse der uberschrift in $k$ glauben schenken, da jungere handschriften oft aus den besten quellen geschöpft hätten, musste doch für unsern speciellen fall erst noch mehr gestutzt und wabrscheinlich gemacht werden, ehe es uns bewegen könnte einem werke einen durch zwei ăltere zeugnisse gestutzten verfassernamen zu nehmen, um auf ein jungeres hin eine der vagen gestalten des Wartburgkrieges dafur einzusetzen. Was den namen Winsbekin betriff, so hat, glaube ich, Haupt allen fruheren fabeleien und märchenhaften hypothesen gegenuber das richtige getroffen, indem er ilun (vorr. XII) fur willkurlich und ungeschickt gesetzt und aus der absicht hervorgegangen erklärte dem ersten gedichte sein gegenstllek auch in der uberschrift gleichzurttcken.

\section{Das handschriftenverbälnis beim Winsbeken.}

Die handschriften, in denen das gedicht des Winsbeken uns uberliefert ist, sind folgende: die Weingarter liederhandschrift B, die Pariser liederhandschrift C, die Berliner Nibelungenhandschrift $I$, die Kolmarer liederhandschrift $k$, eine Gothaer handschrift $g$, eine Basler $K$ und eine Wiener $w$ (in letzteren beiden uur fragmente). Es wird nun im folgenden unsre aufgabe sein die principien fur die textkritik zu gewinnen und das verfahren von Haupt bei seiner herstellung eines gereinigten textes einer nachprufung zu unterziehen. 
Haupt selbst hat ebenso wenig wie Lachmann es geliebt uber seine ansicht vom handschriftenverhältnis mhd. gedichte und seinen daraus etwa gewonnenen kritischen principien in der einleitung in ausfubrlicher weise rechenschaft zu geben. So findet sich in betreff unsres gedichtes nur folgender kurze und ziemlich unbestimmte satz (vorrede VII) 'von diesen handschriften ist $B$ weder die älteste noch die sorgfăligste $\mathrm{zu}$ nennen; sie mag etwas junger sein als I und vor einzelnen versehen baben sich die schreiber von $\mathrm{C}$ und I besser gehlitet: dennoch musste ich sie meiner ausgabe zu grunde legen, da die andern handschriften zwar hier und da richtigeres geben, was mir hoffentlich nirgends entgangen ist, aber im ganzen die uberlieferung, der jene folgt, willkülich verändern.' Ob diese sătze begründet sind, werden wir im folgenden zu untersuchen haben.

Ein kriterium fur die bestimmung der verwantschaft zweier handschriften ist bei unserm gedichte ähnlich wie bei den minnesăngern, die in vielen von einander abweichenden handschriften taberliefert sind, die anzahl und anordnung der strophen, uber die ich im năchsten abschnitt handeln werde. Zeigen sich in diesem punkte bei der natur unsres gedichtes wol gănzlich unbeabsichtigt sich einstellende beziehungen der art, dass sie auf eine nähere verwantschaft $\mathrm{zweier}$ handschriften unter einander hindeuten, so kann man mit ziemlicher sicherheit, falls die vergleichung der lesarten nicht unmittelbar dagegenspricht, zusammenhang oder unabhängigkeit danach feststellell. Absehen muss man bei einer solchen untersuchung naturlich von den făllen, wo sich das fehlen.einer oder meh. rerer strophen aus ausserlichkeiten und fluchtigkeiten, wie z. b. das so hăufige uberspringen auf gleiche worte in einer spăteren strophe erklärt oder auch sonst der verdacht der unechtheit erhoben werden kann. Nach eliminierung solcher falle jedoch wird sich ein in gewissem grade gesichertes resultat ergeben, in wie weit die erhaltenen handschriften oder ibre vorlagen mit einander zusammenhăngen oder nicht. Ich sage, ein in gewissem grade gesichertes resultat, denn mit exacter gewissheit kann in fragen der textkritik sehr oft nicht nach der einen oder andern seite hin entschieden werden: und auch in unserm gedichte gibt es dergleichen fälle genug. 
Die strophenordnung und -anzahl nun ist, wie aus der tabellarischen ubersicht des nächsten capitels mit leichtigkeit ersehen werden kann, bei jeder handschrift eine andre: wir haben, da die fragmentarischen handschriften $\mathrm{K}$ und $\mathrm{w}$ wenig in betracht kommen, weil sie eben wegen ihres fragmentarischen zustandes nicht mit sicherheit beurteilt und rangiert werden können, funf verschiedene gestaltungen der uberlieferung vor uns. Eine vergleichung der lesarten, die ich angestellt habe, fuhrte zu demselben resultate. Es finden sich an keiner stelle $\mathrm{zwischen} \mathrm{zw}$ i handschriften tabereinstimmungen der art und des umfangs, dass sie beide, weil auf eine gemeinsame vorlage zurlickgehend, bei fragen nach kritischer herstellung des textes nur eine stimme haben könnten. Ueberall sehen wir eine wechselnde mannigfaltigkeit der tabereinstimmungen und abweichungen. Man kann schon bei fluchtiger durchmusterung nicht ubersehen, dass zuweilen ubereinstimmungen zwischen handschriften sich finden, die auf den ersten blick eine nahe verwantschaft zu erweisen scheinen: doch sind solche congruenzen einesteils sebr selten, andernteils immer local auf einen sehr kleinen raum beschrănkt; zudem stehen sie auch dann noch an orten, wo kurz vorher und nachher die beiden so eng verwant scheinenden uberlieferungen wider auseinandergehen und uns so jene ubereinstimmungen als zufällig erscheinen lassen. Ich will zum beweise dieser behauptung eine dieser stellen hier anfuhren und zwar diejenige, die mir am meisten auffiel: sie alle einzeln $\mathbf{z u}$ besprechen wurde keinen wert fur unsre untersuchungen haben, da sich solche ubereinstimmungen, wie ich glaube, einzig durch annahme einer unbestimmten anzahl von mittelgliedern wurden erklären lassen, die wir zwischen einer hypothesierten einzigen vorlage und den erbaltenen handschriften anzusetzen hătten, und eben bei ibrer ganz problematischen natur weit mehr dazu angetan sind unser urteil zu truben und auf abwege zu leiten als es zu fördern. Es würden ausserdem doch, selbst wenn wir jene erwähnten mittelglieder statuierten, die erbaltenen uberlieferungen bei ihrer sonstigen verschiedenheit als von einander unabbängig zu gelten haben. Die, ubereinstimmung findet sich in $B$ und $k$ in strophe 50. Beide haben zeile 3 dem und alsô mit der mâze wiget der ubrigen gegenuber wan 
(wanne $\mathrm{k}$ ) er (hat $\mathrm{k}$ ) schanden angesigt (ane gesiget $\mathrm{k}$ ) und lassen darauf die 4. zeile aus; darauf haben beide als 7. zeile eine allen andern feblende: des han ich ie mit willen begert (gert $\mathrm{k})$. Die congruenzen sind frappant und doch düren wir darauf keinerlei schlüsse grüden: denn grade an dieser stelle weichen strophenordnung und -anzahl beider handschriften an meisten von einander ab, ohne dass sich äussere grinde dafür auffinden liessen, und auch die lesarten der nächststehenden in beiden uberlieferten strophen stimmen gerade in charakteristischen dingen nicht mehr zusammen. So hat vielmehr $k$ mehrere lesarten mit $\mathrm{C}$ und I gemein und gerade an für B charakteristischen varianten wie 52,7 das feblende niht, 53,2 gitekeit, 45, 9 fuogen nimmt es keinen teil.

Wir haben also kein recht irgend zwei der handschriften zu einer gruppe zu verbinden. Wären sie nun alle von gleicher absoluter vorzliglichkeit und zuverlässigkeit, so würde sich als oberstes und einziges textkritisches princip einfach der satz ergeben, dass wir die echte lesart immer auf der seite der majorität zu suchen hätten. Da diese bedingung aber kaum je und auch im vorliegenden falle nicht statt hat, so bleibt uns im folgenden noch die frage zu beantworten, in wie weit wir aus der beschaffenheit der einzelnen handschriftlichen uberlieferungen an sich betrachtet textkritische principien gewinnen können. Wir betrachten zu dem zwecke die einzolnen handschriften im hinblick auf ihren wert und den absoluten grad ihrer zuverlăssigkeit.

Von den fragmentarischen handschriften $\mathbf{K}$ und $\mathbf{w}$ können wir im folgenden absehen. $w$ hat in seinen 6 erhaltenen strophen vier sinnentstellende fehler: 2,7 suwercliche $=$ sinnecliche $\mathrm{BCIKgk} ; 2,8$ funden $=\operatorname{swinde}(n) \mathrm{BIKk} ; 6,3$ meinen $=$ nieman $\mathrm{BCIgk} ; 6,5$ schaden = saelden $\mathrm{BCIgk}$. Ueber $\mathrm{K}$ ist nichts besondres $\mathrm{zu}$ bemerken: es zeigt hinneigung zu den lesarten von I, zuweilen auch von $B$, es bleiben also noch $B, C, I, g, k$ ubrig. Diese funf handschriften zerfallen in 2 wei gruppen: eine bessere, die von BCI, und eine schlechtere, die von gk gebildet wird; die handschriften der ersten gruppe sind älter, die der zweiten junger.

In der ersten gruppe ist B bei weitem die unzuverlässigste und schlechteste: sie verdient, wie ich im folgenden zu zeigen 
gedenke, die ehre als grundlage des herzustellenden textes zu dienen, die Haupt ibr erwiesen hat, durchaus nicht. Ich gebe im folgenden zunächst eine ubersicht der hauptsächlichsten offenbaren entstellungen und febler in B. 7, 5 fürbas $=$ ferre baz CIg. 9, 6 so kusche $=$ so ze huse Igk. 9, 9 frünt $=$ tragen $(t)$ CIgk (frument, was Haupt hier aus der lesart von $\mathrm{B}$ durch conjectur gemacht hat, wird schwerlich richtig sein, denn im classischen mhd. steht nach einem imperativ gewöhnlich der conjunctiv; vgl. Paul, Mhd. gramm.2 358). 12,10 der er $=$ daz er CIgk. 13, 3 er dir selben $=$ ez dir saelde CIgk. 16, 7 dienen $=$ dienet $\mathrm{CIgk} .17,3$ rehter $=$ ritter Clgk. 17, 7 spil = zil CIgk. 20,10 teg.enes = siges $\mathrm{CIg}$ (k hat segens, eine lesart, die offenbar mit der von B zusammenhängt; Haupt schreibt degenes: ich glaube, dass siges das echte ist, da der ganze zusammenhang des vergleichs ein wort verlangt, das sowol auf den kampf wie auf das wurfelspiel sich bezieht; zudem wäre das wort degen an sich auffällig). 23, 9 wechseleren = velschelaeren CI. 24, 5 schüsch = schiuz CIgk. 26, 1 swer ze blaiche an sich nimt = swer ze blicke fuoge entnimt Igk. 28, $8 \mathrm{mag}=b a z \mathrm{CIKg}$ (die sonst häufige zusammenstellung von mâc und vriunt kann diese lesart nicht stutzen; zu dem ist nicht einzusehen, wie der $m a \hat{c}$ überhaupt in den zusammenhang des gedankens hineinpasst; offenbar hat Haupt an der construction mit vür nach dem comparativ anstoss genommen; dafur verweise ich auf Paul, Princ. ${ }^{2} 138$, ausserdem macht mich herr professor Paul auf eine stelle in der deutschen grammatik des Laurentius Albertus aufmerksam, wo bogen $M 6^{\mathrm{b}}$ als regelmässige construction angefubrt wird: er ist gelerter für vielen andern). 29, 10 verber $=$ verlür Igk. 32,8 leben $=$ ligen $\mathrm{CIg}$. 35,3 ervraischet $=$ verliuset CIgk. 35,6 verhat $=$ vervat Ig. 36,7 lait $=$ dol CIgk. $38,3.6$ sitten = snitte $\mathrm{CIg} . \quad 40,5$ ruos mur $=$ sur C. 41,7 vermisset $=$ übermisset CIgk. 43,6 guot an = gar ane CIg. 44, 4 gat $=$ stat CIgk. 44, 5 gerne gerat $=$ gerne hete rat CIgk. 45, 9 fuogen $=$ fuoren CIk. 46, 8 und uzgevangen = unuzgevangen $\mathrm{I}$. 51,10 rihtet $=$ richet CI. 52,10 da krenket hin $=$ da schrenket hin Igk. 53, 2 gitekeit = gestricket CIk. 54,7 $e=i e$ CI. 56, 4 reden $=$ raeten CIk. 77,1 iemmer tuon $=$ got ich tuon $\mathrm{I}$, ich tuon C. - Dazu stellen sich folgende auslassungen, bei denen 
nicht selten der sinn des satzes in sein gegenteil umgekehrt erscheint. 3,9 alsô vil. 18,9 ouch. 37,10 er. 38,8 diu. 39,5 nît noch. 48, 10 daz. 49, 8 die. 50, 4 die ganze zoile. 52, 7 niht. 57, 3 vil. 57, 8 hie. 57, 9 er. 60, 9 lobe. Ferner eine reibe von umstellungen: $7,9.29,6.36,5.44,2.57,8.57,9$. $60,4.62,9$. Die meisten der aufgefuhrten varianten kennzeichnen sich auf den ersten blick als mehr oder weniger grobe entstellungen: keine handschrift unsrer ersten gruppe zeigt deren so viele. Wir können sogar unter diesen umständen gar nicht wissen, ob nicht auch andre abweichende lesarten von B, denen man es so auf den ersten blick nicht ansieht, entstellungen in sich bergen.

Ganz anders gestaltet sich das resultat, wenn wir die lesarten von $\mathrm{C}$ durchprufen. Auch hier stossen wir auf viele augenscheinlich neue lesungen: doch sind es meistenteils nicht entstellungen des echten, sondern bewusst vorgenommene correcturen des textes, die die tendenz haben das gedicht $\mathrm{zu}$ glätten und teilweise zu modernisieren. Ich fuhre auch hiervon die wichtigsten auf. 2,7 entstan $=$ verstan Blgk. 2,8 git $=$ wigt $\mathrm{BIgk} ;$ sünden $=$ swinde $\mathrm{BIk} .4,7$ hie halt $=$ behalt BIgk (trotz des dem hie correspondierenden dort in zeile 9 glaube ich nicht, dass $\mathrm{C}$ das richtige bietet). 4,9 behaben = vahen BIgk. 4,10 verhabe - verslahe BIgk. 5,1 sint = ist BIgk. 8,1 hie füge $=($ ge)füge BIgk. 9,2 nuwes vingerlin $=$ minnevingerlin BIgk. 9,4 dast zenge $=z e$ wit ist BIgk. 9,6 niht ze huse - so ze huse Igk. 9, 10 wise = ahte BIg. 15, 2 des = eines BIgk. 15,8 bestrichen = bestricket BIgk. 18, 5 beschach = geschach BIgk. 18,9 eren vil = pris BIgk. 20,8 muoste $=$ muoz BIgk. 21, 10 brust $=$ tjost BIgk. 22,1 zieren $=$ kleiden BIgk. 24, 2 niht $=$ iht BIgk. 28,5 swer $=$ der $\mathrm{BIgk} .29,3$ herze $=\sin \mathrm{Bk}$, tugend $\mathrm{Ig}$. 29, 5 daz ist $=$ ist BIgk. 29,6 dem = swem BIgk. 29,8 den = wen BIgk. 31, 1 wissest $=$ merke BIgk. 31, 3 ellü $=$ ze aller BIgk. 31, 9 den kranken also $=$ die kranckgemuoten BIgk. 31,10 als im - ist $=$ als in - si BIg. 32,9 für ere $=$ verre BIgk. 33,7 und ouch verswigen $=$ und unverswigen Blk. 33,9 an muote $=$ daz muotes BIgk. 34,7 ze selten $=$ ze saelden BIgk. 35, 6 enpfat $=$ vervat $\mathrm{Ig}$. 37,2 tore $=$ narre BIgk. 38,6 der $=$ swer Ig. 39,7 bis $=$ den BIgk. 40,8 snite $=$ schulden $\mathrm{BIg}$. 
40,9 rouch $=$ rum BIgk. 41,6 einer $=$ siner BIgk. 42,4 in gelicher $=$ gelicher BIgk. $43,9.10$ hat $\mathrm{C}$ den ganzen gedanken verăndert. 44,1 ganc $=$ solt gan BIgk. 44,3 schande $=$ schaden BIgk. 44,5 hete gerne - gerne hete Ig. 44,9 besluss - den = sliuss - dinen BIgk. 45, 4 eigen = huobe BIgk. 45,9 valschen $=$ swachen BIgk. 46,7 von $=$ in $\mathrm{BIg}$. 47, 3 ungerihtig $=$ ungereisic B. 47,9 mit eren $=$ in eren BIg. 48,6 wisten $=$ wizzen BIg. 48,8 habe ouch noch $=$ hette noch BIg. 50,8 wesen = wurzelen BIgk. 51, 1 hus han $=$ husere BI. 51,2 eren $=$ tugenden BI. 51,7 sint $=$ werdent $\mathrm{BI} . \quad 51,9$ diu $=\operatorname{disiu} \mathrm{BI} . \quad 52,2$ in $=$ den BIgk. 52,6 geweriu $=$ wariu BIgk. 52, 9 genennet $=$ geheissen BIgk. 52, 10 schüsset hin $=$ schrenket hin Igk. 53,1 bant $=$ ein bant BIk. 53, 5 der $=$ swer BIk. 53,6 finde = findet BIk. 53,9 helfe $=$ helle Blk. 53,10 die not $=$ klam Blk. 54,8 ist $=$ wirt BI. 55, 7 ir $=$ diu BIk. 59, 2 niht nah $=\operatorname{din}$ mit Ik. 59, 3 ist $=$ gat Ik. 60,4 wirt $=$ wurde BIk. 60,6 erkennet $=$ bedenket $\mathrm{BI}$. 62,6 zuo = nach BIk. 63,4 vreude = sünde BIk. 63,6 der sache $=$ dem sacke BIk. 64,5 gerne $=$ lihte BIk. 64,8 ja ist ez niht $=e z$ ist niht BIk. 66, 8 von miner schulde $=$ und dine tugent $\mathrm{Bk} .67,3$ erst bedaht $=$ überdaht Ik. 67,4 do $=$ daz Ik. 69,8 dagen = klagen Ik. 71,9 an $=$ in Ik. 72,5 geschehen = gewesen Ik. 72,9 in $=$ in Ik. 75,6 wildes gie $=$ wilde lief $\mathrm{Ik} .75,8$ leider $=$ kleider $\mathrm{Ik}$. 80, 6 enzien $=$ verzihe $\mathrm{Ik} . \quad 80,8$ füget $=$ fürbas $\mathrm{lk}$.

Bei weitem die beste und zuverlăssigste tuberlieferung liegt uns meiner uberzeugung nach in I vor, der dritten handschrift unsrer ersten gruppe. Sie hat fast keine sinnentstellenden fehler und nur selten auslassungen. Ihr wert ist von Haupt bei seiner textherstellung sicher unterschätzt.

Ueber die handschriften der zweiten gruppe brauche ich nur weniges $\mathrm{zu}$ bemerken. Sie sind sich beide ihrem werte nach so ziemlich gleich: entstellungen, willktirliche ånderungen, grössere und kleinere auslassungen finden sich in $\mathrm{g}$ wie $\mathrm{k}$ in grosser anzahl. Ich brauche sie, da auf sie meistenteils kein wert zu legen ist, hier nicht alle aufzufuhren: sie lassen sich aus dem abdruck von $g$ in Beneckes Beiträgen oder Haupts variantenverzeichnis sowie den angaben meines ersten capitels leicht ersehen. Dazu kommt, dass in $g$, wortber später zu 
handeln sein wird, die echte strophenordnung vollständig taber den haufen geworfen ist.

Gemäss diesen zusammenstellungen und beobachtungen uber die absolute zuverlässigkeit der einzelnen uns vorliegenden handschriften wird in dem widerstreit der lesarten in jedem einzelnen falle $\mathrm{zu}$ entscheiden sein. Die grundlage hat im allgemeinen I zu bilden: da die lesungen dieser handschrift selten allein stehen, haben sie durch die ubereinstimmung noch grössere gewähr der echtheit. Stimmt $\mathrm{C}$ zu I gegen $\mathrm{B}$, so ist die lesart beider unbedingt in den text aufzunehmen, gleichviel ob, was meistens jedoch der fall ist, die handschriften der zweiten gruppe beide oder einzeln bestätigend hinzukommen. Stimmt B zu I gegen C, so hat, wie wir sahen, in den weitaus meisten fällen $\mathrm{C}$ die modernisierende neuerung. Natülich können von diesen allgemeinen sätzen unter umständen auch ausnahmen stattfinden, deren behandlung sich dann aus dem jedesmaligen verhältnis der texte zu einander zu ergeben hat. Eine therlieferung von $g$ und $k$ wird nur in den seltensten fâllen der ubereinstimmung zweier oder aller drei der ersten gruppe gegenuber als echt in den text aufzunehmen sein. Liegt allgemeine verschiedenheit der lesarten vor, so kann naturlich auch auf der seite von $g$ oder $k$ das richtige sein.

III. Die echtheit der strophen beim Winsbeken.

Ich gebe zunächst im folgenden zur bequemeren orientierung eine tabellarische ubersicht der strophenentsprechung. Die erste columne gibt Haupts zahlung.

$\begin{array}{rrrrrrrr} & \text { B } & \text { C } & \text { I } & \text { K } & \text { g } & \text { k } & \text { w } \\ 1 & 1 & 1 & 1 & & 1 & 1 & 1 \\ 2 & 2 & 2 & 2 & 1 & 2 & 2 & 2 \\ 3 & 3 & 3 & 3 & 2 & 7 & 3 & 3 \\ 4 & 4 & 4 & 4 & 3 & 3 & 4 & 4 \\ 5 & 5 & 5 & 5 & 4 & 6 & 5 & 5 \\ 6 & 6 & 6 & 6 & & 5 & 6(7) & 6 \\ 7 & 7 & 7 & 7 & & 19 & 7(8) & \\ 5 & 8 & 8 & 8 & & 26 & 8 & \\ - & & & & & 27 & & \\ 9 & 9 & 9 & 9 & & 13 & 9 & \\ 10 & 10 & 10 & 10 & & 4 & 10 & \end{array}$


ZU WINSBEKE UND WINSBEKIN.

\begin{tabular}{|c|c|c|c|c|c|c|}
\hline & B & $\mathrm{C}$ & I & $\mathbf{K}$ & $\mathbf{g}$ & $\mathbf{k}$ \\
\hline 11 & 11 & 11 & 11 & & 20 & 11 \\
\hline 12 & 12 & 12 & 12 & & 21 & 12 \\
\hline 13 & 13 & 13 & 13 & & 22 & 13 \\
\hline 14 & 14 & 14 & 14 & & 23 & 14 \\
\hline 15 & 15 & 15 & 15 & & 24 & 15 \\
\hline 16 & 16 & 16 & 16 & & 25 & 16 \\
\hline 17 & 17 & 17 & 17 & & 16 & 17 \\
\hline 18 & 18 & 18 & 18 & & & 18 \\
\hline 19 & 19 & 19 & 19 & & 17 & 19 \\
\hline- & & & & & 18 & \\
\hline 20 & 20 & 20 & 20 & & 14 & 20 \\
\hline 21 & 21 & 21 & 21 & & 15 & 21 \\
\hline 22 & 22 & 22 & 22 & 7 & 28 & 22 (1) \\
\hline 23 & 23 & 23 & 23 & & 29 & $23 \quad(2)$ \\
\hline 24 & 24 & 24 & 24 & & 30 & 24 (3) \\
\hline 25 & 25 & 25 & 25 & & 31 & $25 \quad(4)$ \\
\hline 26 & 26 & & 26 & & 32 & $26 \quad(5)$ \\
\hline 27 & 27 & & 27 & & & $27(6)$ \\
\hline 28 & 28 & 27 & 28 & 5 & 34 & 28 (7) \\
\hline 29 & 29 & 29 & 29 & & 35 & $29 \quad(8)$ \\
\hline 30 & 30 & & 30 & & 36 & $30 \quad(9)$ \\
\hline 31 & 31 & 28 & 31 & & 33 & $31(10)$ \\
\hline 32 & 32 & 30 & 32 & & 10 & 32 (11) \\
\hline 33 & 33 & 31 & 33 & & 37 & 33 (12) \\
\hline 34 & 34 & 32 & 34 & & 38 & 34 (13) \\
\hline 35 & 35 & 33 & 35 & & 39 & $35(14)$ \\
\hline 36 & 36 & 34 & 36 & & 40 & $36(15)$ \\
\hline 37 & 37 & 35 & 37 & & 41 & 37 (16) \\
\hline 38 & 38 & 36 & 38 & & 8 & \\
\hline 39 & 39 & 37 & 39 & & 12 & $38(17)$ \\
\hline 40 & 40 & 38 & 40 & & 42 & 39 (18) \\
\hline 41 & 41 & 39 & 41 & & 43 & 40 (19) \\
\hline 42 & 42 & 40 & 42 & & 44 & $41(20)$ \\
\hline 43 & 43 & 41 & 43 & & 45 & \\
\hline - & & & & & 46 & \\
\hline 44 & 44 & 26 & 44 & & 51 & $42(21)$ \\
\hline 45 & 45 & 42 & 45 & & 52 & $43(22)$ \\
\hline 46 & 46 & 43 & 46 & & 48 & \\
\hline 47 & 47 & 44 & 47 & & 50 & \\
\hline 48 & 48 & 45 & 48 & & 49 & \\
\hline 49 & 49 & 46 & 49 & & 11 & \\
\hline 50 & 50 & 47 & 50 & & 9 & $44(23)$ \\
\hline 51 & 51 & 48 & 51 & & & \\
\hline 52 & 52 & 49 & 52 & & 47 & $45(24)$ \\
\hline
\end{tabular}




\begin{tabular}{|c|c|c|c|c|c|}
\hline & B & C & I & $\mathbf{K}$ & $\mathbf{k}$ \\
\hline 53 & 53 & 50 & 53 & & $46(25)$ \\
\hline 54 & 54 & 51 & 54 & & \\
\hline 55 & 55 & 52 & 55 & & $47(26)$ \\
\hline 56 & 56 & 53 & 56 & 6 & $48(27)$ \\
\hline 57 & 57 & 54 & 57 & & $\cdot 1$ \\
\hline 58 & 58 & 55 & 58 & & 2 \\
\hline 59 & & 56 & 59 & & 3 \\
\hline 60 & 59 & 57 & 60 & & 4 \\
\hline 61 & 60 & 58 & 61 & & 5 \\
\hline 62 & 61 & 59 & 62 & & 6 \\
\hline 63 & 62 & 60 & 63 & & 9 \\
\hline- & & & & & 10 \\
\hline 64 & 63 & 61 & 64 & & 11 \\
\hline 65 & 64 & 62 & 65 & & 1 \\
\hline 66 & & 63 & 66 & & 2 \\
\hline 67 & & 64 & 67 & & 3 \\
\hline 68 & & 65 & & & 4 \\
\hline 69 & & 66 & 68 & & 5 \\
\hline 70 & & 67 & 69 & & 6 \\
\hline 71 & & 68 & 70 & & 7 \\
\hline 72 & & 69 & 71 & & 8 \\
\hline 73 & & 70 & 72 & & 9 \\
\hline 74 & & 71 & 73 & & 10 \\
\hline 75 & & 72 & 74 & & 11 \\
\hline 76 & & & 75 & & 12 \\
\hline 77 & 65 & 73 & 76 & & 13 \\
\hline 78 & 66 & 74 & 77 & & 14 \\
\hline 79 & 67 & & & & \\
\hline 80 & & 75 & 78 & & 15 \\
\hline
\end{tabular}

In der vorrede zu seiner ausgabe (s. VIII) hatte Haupt die vermutung aufgestellt, dass das ursprtungliche gedicht des Winsbeken mit strophe 56 abschliesse und alles spätere zusatz eines andern dichters sei, 'fromm, aber albern'. Diese annahme fand allgemeine zustimmung oder doch keinen widerspruch. Sie stutzt sich vor allem auf zwei argumente, ein äusseres und ein inneres. Eines teils nämlich ist der schluss der Winsbekin, wo auch in den letzten strophen drei regeln als besonders vor allen andern wichtig hervorgehoben werden, der 56. strophe des Winsbeken nachgebildet, woraus hervorgeht, dass der dichter der Winsbekin keinen andern schluss des gedichtes vor sich batte als eben die strophe 56. Zweitens 
lehrt eine prifung des gesammtgedichts, dass durch alles, was von strophe 57 an folgt, der inhalt der ersten 56 strophen eigentlich vollkommen zu nichte gemacht wird. Man mag die năhere ausfuhrung dieses arguments in Haupts vorrede nachlesen. Derselbe schreibt die fortsetzung einem geistlichen $\mathrm{zu}$, der als asket das weltleben und die auf vorzugsweise menschlicher basis ruhende moral des ritterlichen lebens hasste und des vaters ermahnungen durch die entgegnung des mönchisch gesinnten sohnes tuberbieten wollte. Gegen diese argumente nun und gegen Haupts hypothese im allgemeinen ist neuerdings Wilken aufgetreten in seinem aufsatz 'zum Winsbeken' Germ. 17, 410. Mit seinen deductionen haben wir uns jetzt auseinanderzusetzen.

Wilken hält Haupts hypothese nicht fur richtig. Gegen Haupts erstes argument, das aus dem schlusse der Winsbekin geschöpft war, bringt er nichts bei: denn auf den einwurf, dass die drei răte beim Winsbeken in einer, bei der Winsbekin in drei strophen abgehandelt werden, will er selbst keinen wert legen (s. 414). Er bemerkt an derselben stelle ganz richtig, dass die nachahmung eine ganz frei gestaltende, keine directe oder sclavische ist: doch will das nichts besagen, wenn nur uberhaupt, wie ausser allem zweifel steht, zugegeben werden muss, dass eine nachahmung vorliegt; ob dieselbe frei oder nicht frei ist, kommt wenig in betracht. Dagegen polemisiert Wilken (s. 415) energischer gegen das aesthetische argument, meiner uberzeugung nach mit ebenso wenig erfolg. Er fuhrt zunächst eine reihe von stellen an, die sich leicht vermehren liessen, wo sich ein 'sittlich-religiöser hintergrund' der lebre des vaters erkennen lasse, ja er schliesst aus dem gebot der hochschätzung der geistlichkeit (strophe 6. 7) und der furcht vor dem verdienten kirchenbann (strophe 53) sogar darauf, dass der ritterliche dichter in den geistlichen stand ubergetreten sei, kommt also schliesslich zu demselben resultate wie v. d. Hagen, der den schluss fur baare historische wahrheit hielt. Wilken nennt die lehre des vaters an den erwähnten stellen ein 'fechten' fir jene besprochenen dinge: schwerlich mit recht; denn die stellen unterscheiden sich in nichts von dem gewöhnlichen ruhigen und gemessenen gange der ermahnung und nichts deutet im entferntesten auf etwaige gegner 
hin, gegen die ein klerikaler fanatiker jene dinge zu verfechten sich vorgenommen hătte. Es sind vielmehr anschauungen ausgesprochen, für die ein fechten gar nicht von nōten war, die den ganzen mittelalter gemein und vertraut waren. Und wer die hăufigen moralisch-religiösen einleitungen mhd. gedichte kennt, dem wird es nicht eigentumlich und individuell, sondern vielmehr selbstverständlich erscheinen, wenn ein didaktisches gedicht auf einen 'sittlich-religiōsen hintergrund' aufgezogen ist. Man lese uber die religiöse grundstimmung unsres gedichtes, die gleichweit von starrem dogmatismus wie von hierarchischem fanatismus absteht, die schöne charakterisierung bei Gervinus nach (Gesch. d. deutsch. dicht. ${ }^{5}$ 2, 3). Die anfuhrung andrer asketischer junglinge und jungfrauen aus der mhd. literatur, wie des Josaphat in Rudolfs Barlaam und der tochter des meiers im armen Heinrich, oder gar des euripideischen Ion (s. 415 anm. 3) kann für unser gedicht nichts beweisen; ebensowenig schliesslich die heranziehung des modernen 'weltschmerzes' oder verweisungen auf Georges psychologie. Gervinus hat vorzliglich die trotz des unvermeidlichen vorwiegens ritterlicher bestrebungen doch im grunde auf echtester humanität ruhende und weit ab von speculationen oder metaphysischen grubeleien auf das praktische leben und seine anspruche gerichtete moral unsres Winsbeken gezeichnet. Haupts hypothese sowie seine argumentation werden durch das, was Wilken dagegen vorgebracht hat, nicbt im mindesten erschuttert.

Wenn wir also auch hier an Haupts hypothese festzuhalten genötigt sind, so findet sich doch in dem, was Wilken gestutzt auf eine randbemerkung in $B$ an ihre stelle setzen wollte, viel brauchbares, was jedoch auch dann vollständig seinen wert behălt, wenn wir nicht mit Wilken die letzten consequenzen ziehen, sondern bei Haupts annahme bleiben. Wilken macht darauf aufmerksam, dass man vielleicht einer bemerkung, die in B am rande der 64. strophe steht, bedeutung beimessen mulsse: sie lautet des vater lêre ze sinem sun hât ende hie. Nach strophe 64 ist ein gewisser abschluss des sinnes stark fublbar. Wilken nun will an dieser stelle den schluss des alten Winsbeken sehen und stitzt sich dabei auf zwei gründe: die 'concinnität der diction', die sich in den 
constant am anfange jeder strophe widerholten anreden sun und vater zeigt, nack strophe 64 jedoch aufhört, und das vorkommen sprichwörtlicher wendungen bis $\mathrm{zu}$ derselben strophe. Was dann von strophe 65 an folgt, soll späterer zụsatz sein. Wilken ist also mit Haupt darin einig, dass er zwei verschiedene teile von verschiedenen verfassern annehmen zu Inüssen glaubt, nur setzt er den trennungstrich an eine andre stelle. Die beobachtungen sind gut und richtig; doch sie beweisen nicht, was sie beweisen sollen: denn man braucht darum nicht anzunehmen, dass strophe 1-64 von einem und demselben dichter geschrieben sind; vielmehr lassen sie sich ebenso gut mit Haupts annahme vereinigen. Auch was dann von der Winsbekin gesagt wird, dass ihre dialogische form wol durch den ansatz des dialogs zwischen vater und sohn (strophe 57-64) als vorbild entstanden sei, beweist nicht die einheitlichkeit der 64 strophen, da wir ihr, wie wir fruher sahen, anderweitig misstrauen mulssen. Ja selbst wenn man Wilken hierin recht gäbe und Haupts erstes argument fallen liesse, was ich jedoch keineswegs befurworten möchte, so wurde zwar als erwiesen zu gelten haben, dass der dichter der Winsbekin von seinem vorbilde die strophen 1-64 vor sich hatte; doch wurde man dann immernoch das aesthetische argument aufrechthalten können und mulssen und es wurde die zufugung von strophe 57-64 nur in etwas frubere zeit binaufrücken.

Nach erwăgung des fur und wider glaube ich, dass wir einesteils bei Haupts hypothese stehen bleiben mlissen, dass von strophe 57 an nicht mehr derselbe dichter spricht, andernteils jedoch den zusatz zu ihr zu machen haben, dass von strophe 65 an ein dritter das wort ergreift.

Wir betrachten nun die verschiedenheiten der strophenzahl in den einzelnen texten und beginnen mit den zwei fortsetzungen des alten gedichts. Wir müssen hier die echtheit der strophen, wenn äussere anhaltspuncte mangeln, möglichst nach denselben principien beurteilen wie oben die echtheit der lesarten. Fur strophe 57-64 stimmen die uberlieferungen im allgemeinen uberein, nur dass $B$ strophe 59 auslässt und $k$ hinter 63 eine neue bietet, die allen andern abgeht. Die 59. strophe wird, weil in CIk bezeugt, wol als echt zu gelten 
baben: dass sie in B fehlt, weiss ich nicht zu erkläreu. Was die neue strophe in $k$ betrifft, die oben im ersten capitel von mir mitgeteilt ist, so ist Bartsch nicht abgeneigt sie für echt zu halten (Kolm. meisterl. s. 82). Ist sie echt, so steht sie jedenfalls hier an falscher stelle. Auch kann sie kaum in der uns tberlieferten gestalt echt sein: das walt in zeile 9 ist eine entstellung, wofür Bartsch site conjiciert hat; ebenso auffăllig ist das iuch der 10. zeile dem sonstigen dir gegenuber. In den kritisch hergestellten text durfte sie jedenfalls nicht aufgenommen werden.

In strophe 68-80 gehen die uberlieferungen weit auseinander. $\mathrm{Zu}$ beachten ist, dass dieser ganzen sthdenklage die beziehungen auf die bestimmte person ganz abgehen mit einziger ausnahme von strophe 80 . Schon Haupt hatte (vorrede VIII) eine von diesen strophen, 'die matt widerholende und schwach bezeugte 68.' fur spăteren zusatz erklärt. Wilken hat dies dann (s. 413) zu beweisen versucht, indem or in ihr gegenuber der 'mit poetischer, ubrigens wol gelungener freiheit' das biblische gleichnis umwandelnden strophe 67 etwas 'gewaltsames und unnötig wider engeren anschluss an dio biblische darstellung erstrebendes' sieht. Man kann dies wol gelten lassen. Wenn auch zu dem schwachen zeugnis von $\mathrm{C}$ jetzt noch $\mathbf{k}$ getreten ist, bin ich doch geneigt die strophe fur spăter eingeschoben zu halten und sie auf rechnung der uberarbeitenden tendenz von $\mathrm{C}$ zu schreiben. Es widerholen sich in 68 einige wendungen der 67: lôn 67,10. 68,4.8; verslâfen (67, 8. 68,9 ; bawen $67,2.68,3$. Eine sichere entscheidung lăsst sich jedoch nicht treffen. In $\mathrm{C}$ fehlt ferner strophe 76, welche auf autorităt von $\mathrm{Ik}$ hin wol als echt zu gelten hat. In $B$ fehlen die strophen $66-76$ und 80 ; die 79. ist allein durch $B$ bezeugt. Wilken hat vermutet, dass die in B uberlieferten strophen den ursprung und den kern der weiterbildung fur das ganze beichtgedicht gebildet hătten, an den sich dann immer mehr und mehr neue strophen angeschlossen bătten. Klare einsicht in die entstehungsgeschichte dieser ganzen schlussstrophen ist nicht zu gewinnen: ich wăre der sonstigen zuverlässigkeit der handschriften nach geneigt die fast vollständig ubereinstimmende strophenzahl in CI, wozu noch $k$ kommt, fur das echte zu halten und in B auslassung anzu- 
nehmen. Dann würde die allein in B stehende 79. strophe als unecht auszuscheiden sein. Vielleicht ist folgende beobachtung dazu angetan dies noch wahrscheinlicher zu machen: im ganzen beichtgedicht spricht eine erste person singularis ich, nur hier in strophe 79 heisst es immer wir. Dazu kommt, dass die strophe ganz aus dem zusammenhang herausfallt. Eine sichere entscheidung nach der einen oder andern seite wage ich nicht zu geben. Klar scheint mir nur, dass wir es hier, indem wir der randbemerkung von $B$ glauben schenken und Wilkens beobachtungen mit in. rechnung ziehen, mit einem neuen, von dem dichter der strophen 57-64 verschiedenen, also dem dritten dichter zu tun haben.

Werfen wir nun noch einen blick auf die verschiedenheiten der strophenuberlieferung in dem alten gedichte (strophe 1-56). Alle strophen des Hauptschen textes in seiner reihenfolge haben nur BI. Auch $k$ folgt dieser reihenfolge, bat jedoch auslassungen: es feblen in $\mathrm{k} 38$, wo vielleicht der gleiche anfang von 38 und 39 dî solt ein taberspringen des schreibers veranlasst hat, $43,46-49,51$ und 54 , welche sămmtlich durch die ubereinstimmung von BCI gesichert sind. - In C finden sich abweichungen von der bisher besprochenen anordnung. 26 und 27 fehlen, sind aber durch BIk gesichert. Auf 25 folgt 44: hier wird eino willkurliche umstellung vorliegen; die $\mathrm{zu}-$ sammenstellung hat ihren grund darin, dass in beiden strophen von der zunge die rede ist, die man im zaum halten soll. Zwischen 28 und 29 ist 31 eingeschoben, wofur ich keinen grund anzugeben weiss. Es fehlt ferner 30, was jedoch durch BIgk vollstandig gesichert ist. - Eine ganz ungeordnete und principlose folge der strophen liegt endlich in $g$ vor. $\mathrm{Zu}$ weilen scheinen verbindende stichwörter die reibenfolge zu erklăren: so erscheint in 3 und 38 diu sêle dort, in 50 und 32 das gleichnis vom vogel, in 26 und 31 merke, was vielleicht fur die zusammenruckung massgebend gewesen ist. Die ordnung von $g$ kann unmöglich die echte sein. Was in $g$ ausgelassen ist, wird durch tbereinstimmung andrer tberlieferungen als echt bezeugt. Am schluss erscheint die handschrift lickenhaft. Drei neue strophen, die $g$ hinter 8,19 und 43 hat, durfen wir, glaube ich, ebenso wenig wie Haupt als echt in den text aufnehmen, obwol sie teilweise nicht ohne geschick gemacht sind. 


\section{JV. Das handschriftenverhältnis und die echtheit der strophen bei der Winsbekin.}

Dass die Winsbekin eine nachahmung des Winsbeken ist und nicht von demselben dichter herruhrt, ist allgemein anerkannt. Schon der ganze stil und die verhältnismässige gedankenarmut zeigen dies: doch finden sich auch beruhrungen mit dem Winsbeken, ubereinstimmende wendungen und ausdrticke, von denen man kaum annehmen kann, dass derselbe dichter sie widerbolt und so gewissermassen sich selbst ausgeschrieben habe. Schon Haupt hat (Vorrede s. XIII) auf einiges derart hingewiesen, wie besonders auf die widerholung des gleichnisses vom vogel (Wkin 9,5; vgl. Wke 32, 1. 50,9) und die helekärppel (Wkin 17,5; vgl. Wke 26,5). Ich fuge noch einige parallelen hinzu: $d a z$ ich in sehe mit vorhten an Wkin $2,4=$ und siht in niht mit vorhten an Wke 5,4; daz ich den wisen wol behage Wkin 12,5, daz wir den wisen wol behagen $44,4=d a z$ si den wîsen wol behage Wke 25,6 ; durch dich verswendet wirt der walt Wkin $13,10=$ swende alsô den walt Wke 20,5; ein wîser man hie vor sô sprach Wkin 16,5 = ez sprach hie vor ein wîser man Wke 5,5 ; du muost diu wange $\hat{u z}$ ougen baden Wkin 17,10= $\hat{a z}$ ougen muoste er wangen baden Wke 64,1 ; an siner werdekeit verzage Wkin $22,10=$ ist ez an werdekeit verzaget Wke 14,8; und mache sich den werden wert Wkin $25,10=$ si machet dich den werden wert Wke 22,6; der wil ze huse unêre laden Wkin 29, 10 $=$ den solta sô ze hâse laden Wke 9,6 ; und grüezen $d \hat{a}$ wir grüezen suln Wkin $44,9=$ und grüeze den dû grüezen solt Wke 39,9. Auf andres komme ich an andrer stelle zurick.

In allen erhaltenen handschriften steht die Winsbekin mit dem Winsbeken zusammen: ausgenommen sind nur die fragmentarischen uberlieferungen $K$ und $w$. Das gedicht findet sich also in $\mathrm{B}, \mathrm{C}, \mathrm{I}, \mathrm{g}$ und $\mathrm{k}$ : allerdings steht auch in $\mathrm{k}$ nur der anfang und in I fehlt der schluss. Während wir nun beim Winsbeken keinerlei handschriftenverwantschaft $\mathrm{zu}$ statuieren vermochten, zeigt sich hier nach lesarten und strophenordnung die engste verbindung 2 wischen $\mathrm{C}$ und g: beide uberlieferungen durfen bei textkritischen fragen nur als eine gelten. Dem gegenuber stehen auf der andern seite $B$ und I, die jedoch 
keineswegs so nabe berubrungen unter einander zeigen als $\mathrm{C}$ und g. $k$ lässt hei seiner fragmentarischen uberlieferung sich nicht sicher zu dem einen oder andern paar stellen: in den erhaltenen strophen steht es gewissermassen mitten inne, allerdings mit einer neigung nach $\mathrm{Cg}$ hin, die sich namentlich in der strophenzahl zeigt; sicheres tiber ibre stellung ist aus den acht strophen, die sie nur bietet, nicht zu gewinnen.

Die absolute zuverlässigkeit der einzelnen handschriften ist ungefähr dieselbe wie beim Winsbeken. B zeigt auch hier eine ganze reihe augenscheinlicher entstellungen, wovon ich die hauptsächlichsten hier anfuhre. 8,6 doch = ouch CIk. 8,7 enbuwet $=$ priset CIgk (Haupt hat die lesart von $\mathrm{B}$ in den text aufgenommen, ich glaube schwerlich mit recht). 9,4 siu wisen worten $=$ diu wisen wort CIgk (I werch). 13,1 vil $=$ wis l. 16,2 da von wil $=d a$ wil I. 21,7 zuo deheime $=$ ze beine CI. 23, 8 in herze sniden $=$ in din herze smiden CIg. 29, 4 balde $=$ walde CIg. $33,5 \mathrm{ir}=$ ein $\mathrm{CIg}$. 34,5 doch = ouch CIg. 36,6 gevern = gern CI (auch hier hat Haupt die lesart von B, die auch $\mathrm{g}$ hat, in den text aufgenommen). Ebenso finden sich wider auslassungen; so fehlt: 2,7 selber. 20,3 wip. 26,1 dir. 33,7 werden. 36,7 sich. 40,3 etlich. I gibt auch hier wider den verhältnismässig besten und reingehaltensten text. Die modernisierende uberarbeitung, die wir fur den Winsbeken bei $\mathrm{C}$ beobachteten, ist hier und ubereinstimmend in $\mathrm{g}$ noch weiter getrieben. Wenn ich alle die augenscheinlichen neuerungen hier auffuhren wollte, musste ich fast das ganze gedicht ausschreiben, denn es ist ein ganz andres geworden. Eine kritische ausgabe wurde am besten beide texte nach oder parallel neben einander bringen, das alte gedicht aus BI und die uberarbeitung aus $\mathrm{Cg}$.

Zur leichteren ubersicht der strophenentsprechungen diene folgende tabelle, in der die erste columne wider Haupts zählung gibt.

$\begin{array}{cccccc} & \text { B } & \text { C } & \text { I } & \text { g } & \text { k } \\ - & & & 1 & & \\ - & & & 2 & & \\ 1 & 1 & 1 & 3 & 1 & 1 \\ 2 & 2 & 2 & 4 & 2 & 2 \\ 3 & & & 5 & & \\ 4 & 3 & 3 & 6 & 3 & 3\end{array}$




\section{LEITZMANN}

$\begin{array}{rrrrrr} & \text { B } & \text { C } & \text { I } & \text { g } & \text { k } \\ \mathbf{5} & \mathbf{4} & \mathbf{4} & \mathbf{7} & \mathbf{4} & \mathbf{4} \\ \mathbf{6} & & \mathbf{5} & \mathbf{8} & \mathbf{5} & \mathbf{5} \\ \mathbf{7} & & \mathbf{6} & \mathbf{9} & \mathbf{6} & \mathbf{6} \\ \mathbf{8} & \mathbf{5} & \mathbf{7} & \mathbf{1 0} & \mathbf{7} & \mathbf{7} \\ \mathbf{9} & \mathbf{6} & \mathbf{8} & 11 & 8 & \mathbf{8} \\ 10 & 7 & 10 & 12 & 10 & \\ 11 & & 11 & 13 & 11 & \\ 12 & & 12 & 14 & 12 & \\ 13 & \mathbf{8} & & 15 & & \\ 14 & 9 & 9 & 16 & 9 & \\ 15 & 10 & & 17 & & \\ 16 & 11 & & 18 & & \\ 17 & & 13 & 19 & 13 & \\ 18 & & 14 & 20 & 14 & \\ 19 & 12 & 15 & 21 & 15 & \\ 20 & 13 & 16 & 22 & 16 & \\ 21 & 14 & 17 & 23 & 17 & \\ 22 & 15 & 18 & 24 & 18 & \\ 23 & 16 & 19 & 25 & 19 & \\ 24 & 17 & 20 & 26 & 20 & \\ 25 & 18 & 21 & 27 & 21 & \\ 26 & 19 & 22 & 28 & 22 & \\ 27 & 20 & 23 & 29 & 23 & \\ 28 & 21 & 24 & 30 & 24 & \\ 29 & 22 & 25 & 31 & 25 & \\ 30 & 23 & 26 & 32 & 26 & \\ 31 & 24 & 27 & 33 & & \\ 32 & & 23 & 34 & 27 & \\ 33 & 25 & 29 & 35 & 28 & \\ 34 & 26 & 30 & 36 & 29 & \\ 35 & 27 & 31 & 37 & 30 & \\ 36 & 28 & 32 & 38 & 31 & \\ 37 & 29 & 33 & 39 & 32 & \\ 38 & 30 & 34 & & 33 & \\ 39 & 31 & 35 & & 34 & \\ 40 & 32 & 36 & & 35 & \\ 41 & 33 & 37 & & 36 & \\ 42 & 34 & 38 & & 37 & \\ 43 & 35 & 39 & & 38 & \\ 44 & 36 & & & & \\ 45 & 37 & & & & \\ & & & & & \end{array}$

I gibt den vollständigsten text, nur dass der schluss fehlt. Die zwei strophen, die I vor dem anfang des gedichts hat, 
können, wenn sie uberhaupt echt sind, jedenfalls nicht an dieser stelle vor dem epischen eingang steben. Die 3. stropbe, die nur in I uberliefert ist, hat nichts anstössiges. B zeigt viele auslassungen von strophen, deren echtheit jedoch durch Clgk resp. CIg vollständig gesichert ist. Einen erklärungsgrund für die auslassungen weiss ich hier ebenso wenig anzugeben wie oben bei der fortsetzung des Winsbeken: nur scheint mir klar, dass das fehlen von 32 dupch den gleichen anfang diu huote in 31 und 32 und dadurch veranlasstes uberspringen des schreibers hervorgerufen ist. Die tuberlieferung in Cgk ist ganz einheitlich: denn in $\mathrm{g}$ fehlt die 31. strophe nur durch denselben zufall wie in B die 32. Die umstellung von 14 zwischen 9 , und 10 ist neuerung (vielleicht durch das zweimalige die sinne veranlasst?). Die strophen 13,15 und 16 sind durch BI als echt gesichert: ibre auslassung könnte aus dem gleichen schlusswort $\sin$ in 12 und 16 erklärt werden. 44 und 45 , die auch in $O g$ fehlen, sind zwar nur durch $B$ bezeugt, aber inhaltlich notwendig.

\section{Winsbeke und Wigalois.}

Anhangsweise bespreche ich noch eine beziehung, die man zwischen dem Winsbeken und Wirnt von Grafenbergs Wigalois hat sehen wollen. Der erste, der dies tat, war Pfeiffer. In seiner ausgabe des Wigalois sagt er (vorrede s. XVII): 'aus einigen stellen glaube ich schliessen zu durfen, der Winsbeke, Wirnts landsmann, habe den Wigalois gekannt; ja mir scheint sogar, als ob dessen gedichte die väterlichen lehren zu grunde lägen, die Gawein am schlusse seinem sohne gibt. Jedenfalls herrscht zwischen beiden eine merkwurdige tbereinstimmung, die nicht wol bloss zufällig sein kann.' Leider hat er diese stellen nicht näher angegeben, so dass man diese 'merkwürdigen ubereinstimmungen' nicht controlieren kann. Die von Haupt in den anmerkungen zu 42,9 und 69,7 citierten kann er nicht meinen, denn sie enthalten nichts beweiskrăftiges. Auch ein nochmaliges durchlesen des Wigalois hat mich keine einzige stelle derart finden lassen. Pfeiffer stlutzt. sich also vor allem auf die ermahnung des Gawein an seinen sohn Wigalois am schlusse des gedichts. Die verse lauten $(293,17)$ : 
got hât sîn wunder und sîne gnâde an iu getân. ir sult im wesen undertîn und minnet in herzeliche. der sinne sît ir rîche, des guotes und der êren: daz sult ir allez kêren, swâ ir muget, nach sînem gebot. swer herzenliche minnet got, der ist behalten hie und dort. sun, nû merket disiu wort und behaltet diu âne missetât: daz ist mînes herzen rât. sit bescheiden an allen dingen und lât niht verdringen die jugent iuwer sinne. der iuch mit triuwen minne, an den sult ir iuch lâzen und boeses schimphes mâzen. vernemet armer liute klage und büezet ir kumber alle tage. sit gewizzen unde guot. den vînden traget hôhen muot, den vriunden sît geselleclîch und milte: sô werdet ir lobes rîch. bietet den gesten êre nâch iuwer gewizzen lêre. sît dem gehülfec unde guot, der inwern willen gerne tuot: disem râte volget nâch. lât iuwern zorn niht wesen gâch. traget scham ob allen inwern siten.

Ich finde in dieser ganzen stelle nicht eine einzige wendung, die mit dem Winsbeken so ubereinstimmte, dass wir eine benutzung des einen durch den andern annehmen mussten. Welcher dann ubrigens der benutzende und welcher der benutzte sein wurde, ist auch nicht ohne weiteres klar: Pfeiffer nimmt, ich sehe nicht ein warum, priorität des Wigalois an. Ich glaube, dass Pfeiffer durch die allgemeine abereinstimmung des motivs und vielleicht nicht zum wenigsten durch die nachbarschaft der städte Winsbach und Gräfenberg, auf die er in der anmerkung zu der oben citierten stelle hinweist, auf seine hypothese gefuhrt wurde, die ubrigens mit aller schuldigen reserve und eben nur als vermutung gegeben ist.

Nicht mit derselben vorsicht sind die späteren literarhistoriker vorgegangen, bei denen sogar die hypothese als bewiesene wahrheit figuriert und zur chronologischen bestimmung verwertet wird. So gibt Wackernagel (12,346 anm. 13) ganz definitiv rom Winsbeken an: 'nach Wirnts Wigalois'. Bei Gervinus (51,574) heisst es: 'wo er (Wirnt) den Gawein seinem sohne gute lehren erteilen lässt, redet er .... in den ansichten und in einzelnen ausdrucken, die in den lehren des Winsbeke .... widerkehren'; was ferner von Gervinus behauptet wird, dass in der stelle Wig. 77, 9 uber das verliegen sich anklänge an den Winsbeke fänden, kann ich ebenso wenig zugeben. Auch Goedeke sieht 'auffallende ubereinstimmung' und 'nahe be- 
rlubrung' (Grundr. 12, 101. 162); ähnlich Koberstein $\left(1^{6}, 266\right.$ anm. 26).

Ich glaube, dass wir bei vorurteilsfreier betrachtung dieser hypothese nicht beistimmen können und dass sie vor allem in den literaturgeschichten nicht als bewiesene sache vorgetragen werden darf. Soll ich eine vermutung äussern, so will es mir scheinen, als ob so wol fur Wirnt als fur den Winsbeken das vorbild dieser ritterlichen ermahnung die classische verwendung dieses motivs bei Wolfram gewesen sei, der rat des Gurnemanz an Parzival. Dass der Winsbeke gerade diese partie des Parzival gekannt hat, dafur gedenke ich an andrer stelle noch belege beizubringen.

FREIBURG, 18. februar 1887.

ALBERT LEITZMANN. 